in vivo $35: 533-539(2021)$

doi:10.21873/invivo.12288

\title{
Pathological Examination of the Late Embryonic Heart Using the Same 4-chamber and 3-vessel Planes Used in Fetal Echocardiography
}

\author{
CĂTĂLIN GABRIEL HERGHELEGIU ${ }^{1}$, ADRIAN NEACȘU ${ }^{1}$, RALUCA CHIRCURESCU ${ }^{2}$, DORU HERGHELEGIU ${ }^{1}$, \\ SILVIU CRISTIAN VOINEA ${ }^{3}$, CAMELIA DIACONU ${ }^{4,5}$, OVIDIU STIRU $^{6,7}$, CORNEL SAVU $^{8,9}$, \\ ALEXANDRU FILIPESCU ${ }^{1,10}$, IRINA BALESCU ${ }^{11}$, NICOLAE BACALBASA ${ }^{1,12,13}$ and NICOLAE SUCIU ${ }^{1,14}$ \\ ${ }^{1}$ Department of Obstetrics and Gynecology, \\ "Carol Davila" University of Medicine and Pharmacy, Bucharest, Romania; \\ ${ }^{2}$ Department of Pathology, "Polizu" Clinical Hospital, INSMC "Alessandrescu-Rusescu", Bucharest, Romania; \\ ${ }^{3}$ Department of Surgical Oncology, "Carol Davila" University of Medicine and Pharmacy, Bucharest, Romania; \\ ${ }^{4}$ Department of Internal Medicine, "Carol Davila" University of Medicine and Pharmacy, Bucharest, Romania; \\ ${ }^{5}$ Department of Internal Medicine, Clinical Emergency Hospital of Bucharest, Bucharest, Romania; \\ ${ }^{6}$ Department of Cardiovascular Surgery, \\ "Carol Davila" University of Medicine and Pharmacy, Bucharest, Romania; \\ ${ }^{7}$ Department of Cardiovascular Surgery, \\ "Prof. Dr. C.C. Iliescu" Emergency Institute for Cardiovascular Diseases, Bucharest, Romania; \\ ${ }^{8}$ Department of Thoracic Surgery, "Carol Davila" University of Medicine and Pharmacy, Bucharest, Romania; \\ ${ }^{9}$ Department of Thoracic Surgery, "Marius Nasta” National Institute of Pneumology, Bucharest, Romania; \\ ${ }^{10}$ Department of Obstetrics and Gynecology, "Elias" Emergency Hospital, Bucharest, Romania; \\ ${ }^{11}$ Department of Visceral Surgery, "Ponderas" Academic Hospital, Bucharest, Romania; \\ ${ }^{12}$ Department of Visceral Surgery, Center of Excellence in Translational Medicine, \\ "Fundeni" Clinical Institute, Bucharest, Romania; \\ ${ }^{13}$ Department of Obstetrics and Gynecology, "I. Cantacuzino" Clinical Hospital, Bucharest, Romania; \\ ${ }^{14}$ Department of Obstetrics and Gynecology, "Polizu” Clinical Hospital, \\ INSMC "Alessandrescu-Rusescu”, Bucharest, Romania
}

\begin{abstract}
Background/Aim: The incidence of early pregnancy loss widely varies according to age, being considerably higher in older women. Severe congenital malformations play an important role in pregnancy loss, having a high risk of recurrence. Congenital heart defects are the most common congenital abnormalities, thus the diagnosis of such malformations in aborted embryos is important for establishing both a possible cause for pregnancy loss and for correctly counseling the parents. Pathologic examination of
\end{abstract}

This article is freely accessible online.

Correspondence to: Nicolae Bacalbasa, Carol Davila University of Medicine and Pharmacy, Bucharest, Romania. Tel: +40 723540426, e-mail: nicolae_bacalbasa@yahoo.ro

Key Words: 4-chamber, 3-vessel, congenital heart defect, first trimester, ultrasound. the heart that is only a few millimeters in size, is very challenging. Patients and Methods: A pathologic examination protocol using transverse microscopic sections at the level of the 4-chamber and 3-vessel planes is proposed for heart evaluation. Results: Two 9-10 gestational weeks embryos were microscopically examined using transverse slides of the thorax. The 4-chamber and 3-vessel slides were analyzed and compared to 11-13 weeks ultrasound images of the 4-chamber and 3-vessel views from 10 cases. The pathologic examination provided a detailed view of the ventricles, atria and great vessels, sometime surpassing even the ultrasound examination that was performed at a later gestational age. Conclusion: We consider our proposed pathologic examination protocol feasible for evaluating normal heart structures and ruling out severe congenital heart disease.

The incidence of early pregnancy loss is between $10-15 \%$, with a significant variation according to age, being considerably higher in older women $(1,2)$. The exact causes 
of early pregnancy loss are still debated, but many studies suggest that severe congenital malformations may play an important role $(3,4)$. Furthermore, infants of women with three or more pregnancy losses have almost double the risk of having serious birth defects, compared to infants of women with no prior pregnancy loss (5). As congenital heart defects (CHD) represent the most common congenital abnormality, it makes sense to suspect that they may be linked to a significant proportion of early pregnancy loses $(6,7)$. A number of studies confirm this theory and report that the incidence of CHD in aborted fetuses and embryos is much higher than in liveborn (8). Also, they indicate that the incidence of CHD is higher in the case of early spontaneous abortions, up to $39.5 \%$ for pregnancies under 10 weeks (9). Still the incidence reported by different studies varies widely, especially for small gestational age abortions. One explanation might be that the pathology study of embryos and small fetuses is very difficult and time consuming. Also, for dissecting under the microscope delicate anatomical structures, like the heart that is only a few millimeters in size, specialized personnel with dedicated training is needed. Thus, especially in the case of early first trimester abortions, pathologic examination usually consists of gross and microscopic examination of the embryo and placental tissue, with the main goal being microscopic documentation of chorionic villi. The development of the heart is mostly complete by 7-8 weeks of gestation so if a cardiac anomaly does exist, it could be detectable from this age during pathology examination (10).

For this reason, in order to increase the detection rate of CHD in early pregnancy loses, we tried to come up with a simple, yet efficient method for pathologic evaluation of the embryonic heart at 8-10 weeks of gestation, that allows assessment of the normal development of the main cardiac and thoracic structures and can rule out severe CHD. Also, we attempted to compare the pathology images with ultrasound images obtained at 11-13 weeks of gestation, when early cardiac screening takes place.

\section{Patients and Methods}

Plane selection. In our pathologic examination protocol, we decided to break down the embryonic heart into two basic components, the cardiac chambers and the great arteries. For each component, one easily obtainable transverse section was selected, on which the cardiac anatomy could be studied. For evaluating the atria and ventricles the choice was simple, the 4-chamber view. This is the first and most widely used cardiac section in ultrasound for CHD screening $(11,12)$. Deciding what section to use for evaluating the great arteries was not so easy; as the aorta and pulmonary artery have a complex spatial relationship and their emergence from the ventricles are in different, slightly oblique planes. In the end the decision was made to use the 3 -vessel view as it permits an objective way of assessing the normal morphology of the upper mediastinum and the great arteries $(11,13,14)$. Both sections are transverse planes of the thorax and are borrowed from the ultrasound evaluation of the fetal heart in both first and second trimester $(15,16)$. Other views used in fetal echocardiography, like the left and right ventricular outflow tract views, may be useful in diagnosing certain types of CHD like Tetralogy of Fallot, transposition of the great arteries or double outlet right ventricle (17, 18), but these planes are slightly oblique and hard to be obtained during first trimester ultrasound evaluation, not to mention during pathologic examinations of embryos.

Pathologic examination. Two intact embryos from spontaneous abortions were included in the study for pathologic examination. The gestational ages were 9-10 weeks, corresponding to a 7-8 weeks embryo and it was calculated using the crown-rump length, measured by ultrasound before the abortion. Both cases were fixed in $10 \%$ formalin solution for 10-14 days. After that, the embryos were embedded in paraffin blocks, oriented such as successive thin $15 \mu \mathrm{m}$ thick transverse section of the thorax could be cut with the microtome. The sections were put on microscope slides and Hematoxylin-Eosin staining was used to better highlight the different tissues and structures. The sections through the heart resembling the 4-chamber view and through the upper mediastinum with the great arteries resembling the 3-vessel view were selected and images captured using a dedicated acquisition camera.

Ultrasound examination. A total of ten fetuses with a gestational age between 11 to 13 weeks were included in the study and scanned using a GE Voluson E10 machine. To obtain the best quality images possible, transvaginal sonography with a high frequency transducer (RIC5-9D) was used in the study, as it allows for higher-resolution imaging of the heart in early gestation (19). Both B-mode and Color Doppler Flow were used to examine the heart and a maximum examination time of 30 minutes was allocated for each case.

Structures evaluated. The pathology and ultrasound images were analyzed and we tried to identify 15 main anatomical structures: right and left atria, right and left ventricles, tricuspid and mitral valves, ascending and descending aorta, pulmonary artery, superior vena cava, lungs, trachea, esophagus, vertebra, ribs, sternum, spinal cord. The pathology images were reviewed by a pathologist alongside an obstetrician. The ultrasound images were reviewed by two obstetricians.

\section{Results}

The 4-chamber plane. In all cases, both on pathology and ultrasound images, we were able to identify the heart's position, size and axis, relative to the thorax. Also, the lungs were visible, but in the ultrasound images little details could be observed, as their grey scale appearance was quite difficult to be differentiated from the appearance of the liver or surrounding structures.

In the pathology images of the two 9-10 weeks embryos, the right and left ventricles were easily identified, with a clear, thick, interventricular septum separating them (Figure 1A). The left ventricle, being slightly longer in length than the right one, forms the apex of the heart. At the apex of the 

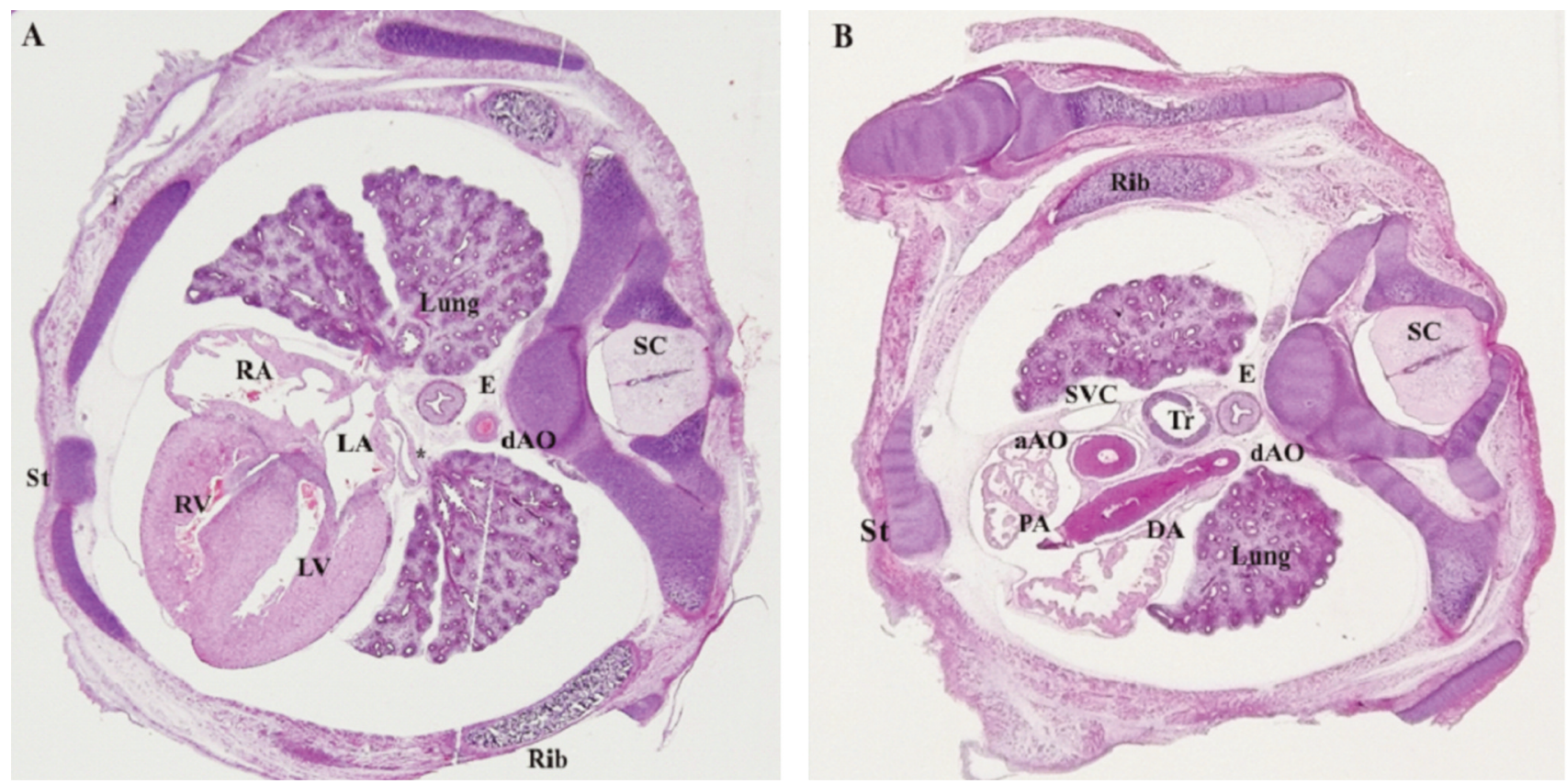

Figure 1. Pathology examination of the 4-chamber (A) and 3-vessel planes (B). RV: Right ventricle, LV: left ventricle, aAO: anscending aorta, dAO: descending aorta, AOc: aortic arch, DA: ductus arteriosus, SVC: superior vena cava, Pv: pulmonary vein, E: esophagus, Tr: trachea, St: sternum, SC: spinal cord.

right ventricle, the moderator band is visible. The atria, with their thin walls, were seen but it was nearly impossible to visualize the whole interatrial septum and foramen ovale flap. Also, just one pulmonary vein opening in the left atrium was seen in one of the cases. The mitral and tricuspid valves were partially identifiable and it was hard to make any comments about their function. The descending aorta was visible posteriorly and to the left, in front of the vertebral body. The esophagus was present to the right and in front of the descending aorta and vertebral body. The vertebra, a rib on each side and the sternum were all seen in great detail. Also, the spinal cord was clearly visible inside the vertebral canal.

In the ultrasound B-mode images of the 11-13 weeks fetuses, it was possible to identify the ventricles, interventricular septum and atria in all cases (Figure 2A). The left ventricle appeared longer in length, while at the apex of the right ventricle, the moderator band was present, making it appear shorter. For viewing the interventricular septum, with its thin membranous portion, a slightly transvers orientation of the heart, with the apex pointing at 3 or 9 o'clock, was helpful (Figure 2B). The atrioventricular valves were distinguishable in 8 cases and only in an apical orientation of the heart. The pulmonary veins were not visible. With the aid of color Doppler, it was much easier to see the ventricles with 2 distinct inflows coming from the atria, through the tricuspid and mitral valves (Figure 3A). In all cases the flow was anterograde and nonturbulent, without antialiasing. By lowering the PRF it was possible to identify at least one pulmonary vein on each side in 6 of the cases, draining in to the left atrium (Figure 3B). The descending aorta was visible in only 7 of the cases and only with the aid of color Doppler and a low PRF setting. The ribs, one on each side, and the ossification centers at the level of the vertebra were visible, but had a relatively blurry contour. The sternum, esophagus and spinal cord were not visible.

The 3-vessel plane. On the pathology slides, a detailed image of the upper mediastinum was obtained (Figure 1B). The 3 vessels represented by the superior vena cava, ascending aorta and pulmonary artery were seen. The superior vena cava, with its thin walls, is the smallest vessel in size and positioned posteriorly and to the right. Next to it to the left, the ascending aorta with ticker arterial walls is larger in diameter and position further anteriorly. The pulmonary artery is the most anterior and leftward vessel, having the diameter roughly the size of the aortic one or slightly bigger. It continues posteriorly with the ductus arteriosus arch, reaching into the descending aorta. The aorta and ductus arteriosus arch somewhat resemble the "Y" sign described in echocardiography. Even if a more cephalad plane is selected, it was not possible to view both the aortic arch and the ductal arch converging and forming the " $\mathrm{V}$ " sign described in echocardiography. The trachea and esophagus were clearly visible, located posteriorly, in front of the vertebral body and 

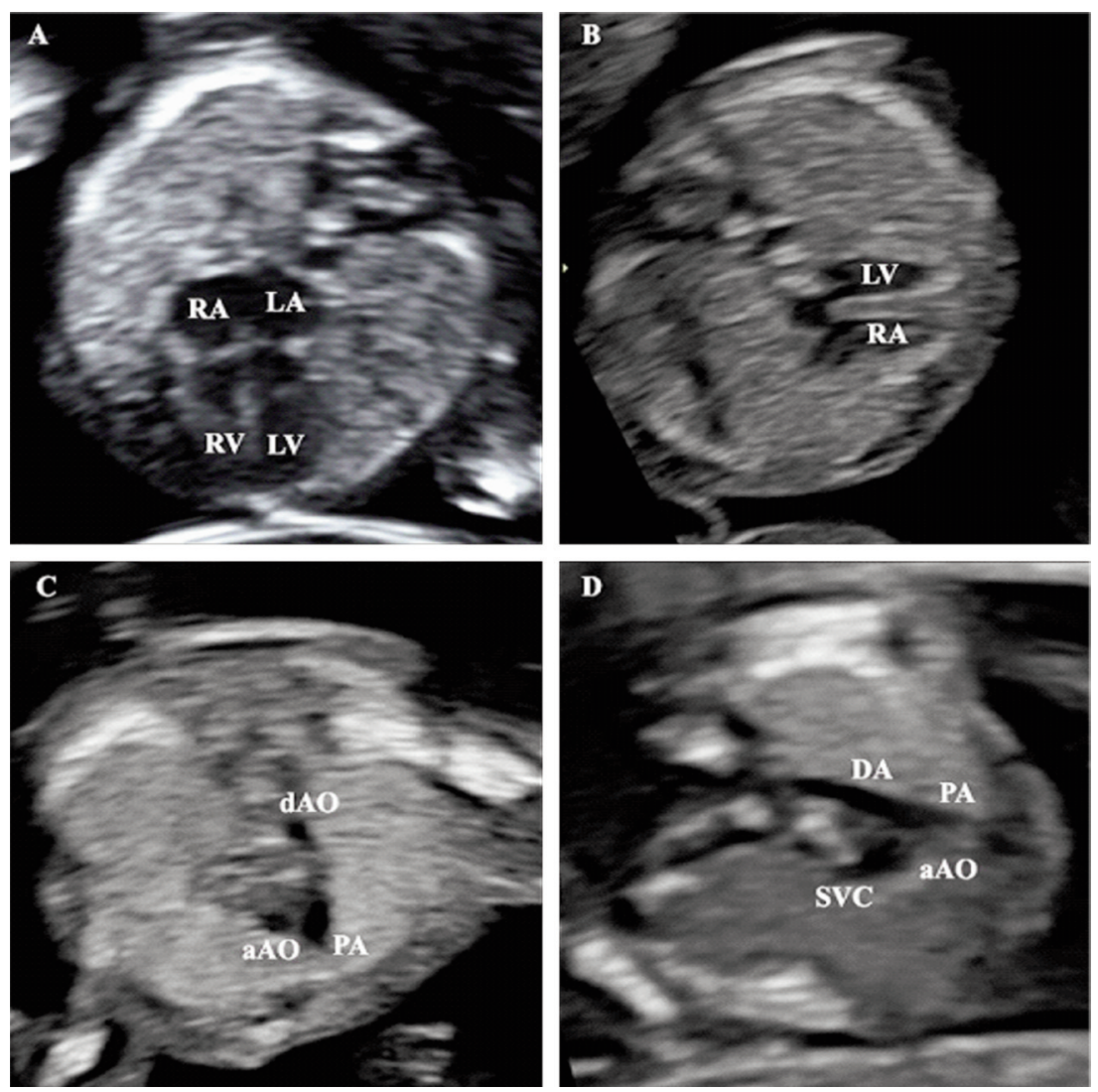

Figure 2. Ultrasound B-mode examination of the 4-chamber (A, B) and 3-vessel (C, D) planes. RV: Right ventricle, LV: left ventricle, aAO: anscending aorta, dAO: descending aorta, AOc: aortic arch, DA: ductus arteriosus, SVC: superior vena cava, Pv: pulmonary vein.

to the right of the aortic and ductus arteriosus arches. The lungs with their typical histological appearance were visible, surrounding on both sides the mediastinum. The vertebra, ribs and sternum were also visible in great detail, as well as the spinal cord, clearly visible inside the vertebral canal.

Using B-mode, the 3 vessels were barely visible, with the superior vena cava being identified in just 4 of the cases. Little information regarding the size, course and alignment of the 3 vessels could be gathered, as the aorta and pulmonary artery had a somewhat imprecise contour (Figure $2 \mathrm{C}$ and $\mathrm{D})$. Changing the insonation angle and choosing an apical 3-vessel view did not help, as the aorta and pulmonary artery appeared even smaller. The mediastinum and lungs were poorly defined between the hyperechoic ribs and ossification centers of the vertebral body posteriorly. By adding color Doppler, the level of detail increased remarkably, especially for the great arteries. In all cases, the aorta and pulmonary artery, continuing posteriorly with the ductus arteriosus arch were clearly seen, forming the characteristic "Y" sign (Figure 3C). Furthermore, the normal relationship between the two vessels was more evident, with the pulmonary artery being slightly larger and more anterior than the aorta. If a slightly higher plane was chosen, then the aortic arch was also visible, converging with the ductus arteriosus arch and forming the "V" sign (Figure 3D). Though the trachea was hard to be visualized, the straight course of the aortic arch suggests that it follows a normal path to the left of the trachea. Also, by studying the color pattern, it was possible to obtain information about the flow of blood across the two arteries. Normal, high velocity, anterograde, nonturbulent, systolic flow was observed in all cases. By lowering the PRF it was possible to view the superior vena cava in 3 cases. The sternum, trachea, esophagus and spinal cord were not visible.

\section{Discussion}

Using the proposed protocol, the pathologic examination of the 9-10 weeks embryos offered highly detailed images of the 4-chamber and 3-vessel planes, allowing all of the 15 main anatomical structures investigated to be identified and surpassing the ultrasound B-mode examination, although the said ultrasound examination was performed at a later gestational age of 11-13 weeks. For cardiac assessment and 

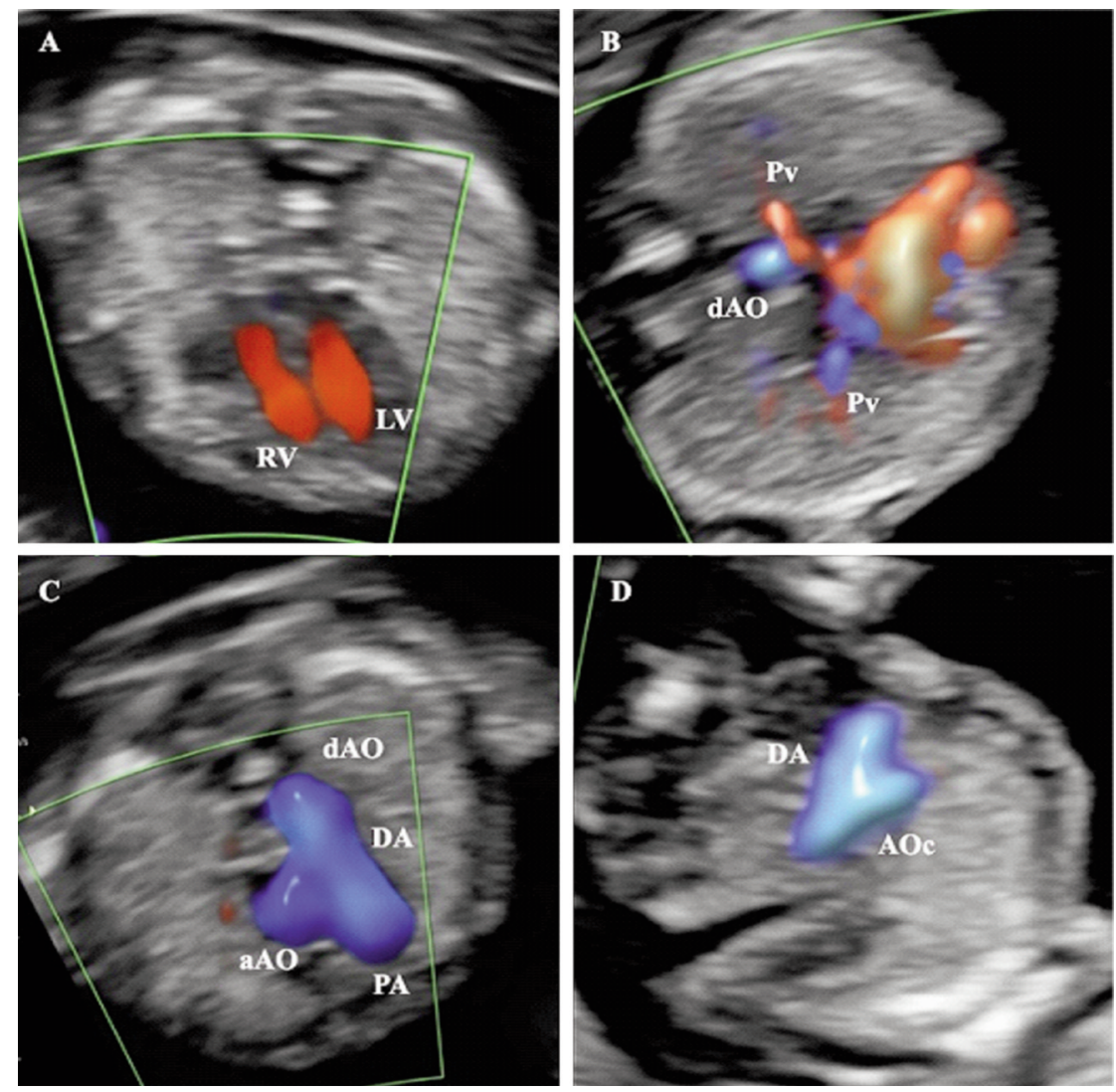

Figure 3. Ultrasound Color Doppler examination of the 4-chamber $(A, B)$ and 3-vessel $(C, D)$ planes. RV: Right ventricle, LV: left ventricle, aAO: anscending aorta, dAO: descending aorta, AOc: aortic arch, DA: ductus arteriosus, PA: pulmonary artery.

CHD screening, both pathologic examination and ultrasound examination with Color Doppler offered similar results, allowing the evaluation of the main cardiac structures and great vessels.

Cardiac structures were easy to be assessed in the pathology images of the 4-chamber plane, as the ventricles, atria, interventricular septum were all clearly visible and well defined (Figure 1A). In comparison, the B-mode ultrasound evaluation provided a poorer level of detail and it was highly dependent on fetal position and heart orientation in relationship with the ultrasound transducer. The mitral and tricuspid valves were visible on both pathology and ultrasound images, but the level of detail was low. By adding Color Doppler to the ultrasound examination, it was simple to perform a hemodynamic evaluation of the heart and it offered indirect but valuable information about the structure of the 4 chambers (presence of a right and left ventricle separated by a septum and two atria) and about the function of the atrioventricular valves (Figure 2A). Also, by using Color Doppler, it was possible to view small vessels, like the pulmonary veins, on each side of the left atrium (Figure 2B).
Pathology images of the 3-vessel plane provide information about the anatomy of the great arteries and their relationship with the surrounding anatomical structures (Figure 1B). From right to left, the superior vena cava, ascending aorta and pulmonary artery were seen in the upper mediastinum. The ductus arteriosus arch and aortic arch were visible, but it was not possible to obtain a slice with both vessels converging towards the descending aorta and passing leftward of the trachea, as seen during ultrasound examination. The relative sizes of the aorta and pulmonary artery could be easily evaluated in the ascending portions of the two. Ultrasound Bmode evaluation offered poor quality images of the 3-vessel plane. The use of Color Doppler allowed a detailed visualization of the aorta and pulmonary artery, forming the "Y" and " $\mathrm{V}$ " sign, depending on the level of the plane (Figure 3C and D). The normal Color Doppler flow pattern observed at the level of the 3-vessel plane offers indirect information about the aortic and pulmonary valves, as valve stenosis or insufficiency would have altered the flow, appearing turbulent, retrograde, small in size or even absent in the corresponding vessel.

Besides important details about the cardiac structures, the pathology slides of the 4-chamber and 3-vessel planes also 
offered excellent, detailed images of the lungs, trachea, esophagus, descending aorta, vertebra, ribs, sternum and spinal cord, (Figure 1A and B) that were not clearly visible on the B-mode ultrasound images.

One of the biggest advantages of this pathologic examination protocol is that it permits the visualization of the heart and great vessels at a very small gestational age, 910 weeks, and it allows the assessment of the normal cardiac and thoracic structures. At this gestational age, it is extremely difficult and time consuming to perform surgical dissection of the embryo and visualize the cardiac anatomy. Another advantage is that the microscope slides that are obtained can be stored for a long period of time and can be later reviewed, if more anatomical details are needed. Also, the microscope slides can be independently reviewed by more pathologists and/or obstetricians if there are any doubts about the diagnosis. By using this pathologic examination protocol, major CHD such as hypoplastic left heart syndrome, with a poor prognostic, that are diagnosed or suspected by ultrasound can be confirmed post abortion (20). The main drawback/disadvantage of the pathology examination protocol we proposed is the number of transverse sections that are necessary to be made through the embryo's thorax. The higher the number of slices, the higher was the chance for obtaining a slice at the correct level and more accurate details. Also, for this protocol to work and obtain transverse planes, a careful orientation of the specimen must be made before embedding it into paraffin.

\section{Conclusion}

Pathologic examination of the late embryonic heart at 9-10 weeks of gestation, using transverse microscopic sections at the level of the 4-chamber and 3-vessel planes provides valuable information about the atria, ventricles and great arteries. As a screening method for CHD, the proposed pathology examination protocol must be able to provide at least the same level of information as the current 11-13 weeks ultrasound screening protocol using Color Doppler, as well as achieve 2 goals $(16,21-23)$. First of all, in the 4-chamber plane, it must be able to assess the position, orientation and size of the heart, as well as confirm the presence of two normally formed ventricles. Secondly, in the 3-vessel plane, it must be able to assess the presence, position, size and course of the aorta and pulmonary artery. We consider that the pathology protocol we proposed, achieves both goals and thus it can be used as an effective method of detecting CHD in the case of early abortions. Furthermore, although performed at a smaller gestational age, the pathology examination provided more detailed images than the 11-13 weeks B-mode ultrasound examination, allowing accurate visualization of important non-cardiac structures, such as the trachea, esophagus and spinal cord.

\section{Conflicts of Interest}

The Authors declare that they have no conflicts of interest.

\section{Authors' Contributions}

$\mathrm{AN}, \mathrm{DH}, \mathrm{CD}, \mathrm{OS}, \mathrm{CS}, \mathrm{AF}$ performed the imaging analysis of the embryos; NB, IB, AF, SCV, NS reviewed literature data; RC performed tha pathological analysis of the specimens, IB, NB, $\mathrm{CGH}, \mathrm{RC}$ prepared the draft of the manuscript; NB, NS reviewed the final version of the manuscript. The Authors read and approved the final version of the manuscript.

\section{References}

1 Larsen EC, Christiansen OB, Kolte AM and Macklon N: New insights into mechanisms behind miscarriage. BMC Med 11: 154, 2013. PMID: 23803387. DOI: 10.1186/1741-7015-11-154

2 Wang X, Chen C, Wang L, Chen D, Guang W and French J: Conception, early pregnancy loss, and time to clinical pregnancy: a population-based prospective study. Fertil Steril 79(3): 577-584, 2003. PMID: 12620443. DOI: 10.1016/s00150282(02)04694-0

3 Yang J, Wang Y, Wang XY, Zhao YY, Wang J and Zhao YY: Adverse pregnancy outcomes of patients with history of firsttrimester recurrent spontaneous abortion. Biomed Res Int 2017: 4359424, 2017. PMID: 28798930. DOI: 10.1155/2017/4359424

4 Ionescu CA, Vladareanu S, Tudorache S, Ples L, Herghelegiu CG, Neacsu A, Navolan D, Dragan I and Oprescu DN: The wide spectrum of ultrasound diagnosis of holoprosencephaly. Med Ultrason 21: 163-169, 2019. DOI: 10.11152/mu-1614

5 Khoury MJ and Erickson JD: Recurrent pregnancy loss as an indicator for increased risk of birth defects: a population-based case-control study. Paediatr Perinat Epidemiol 7(4): 404-416, 1993. PMID: 8290380. DOI: 10.1111/j.1365-3016.1993.tb00422.x

6 Donofrio MT, Moon-Grady AJ, Hornberger LK, Copel JA, Sklansky MS, Abuhamad A, Cuneo BF, Huhta JC, Jonas RA, Krishnan A, Lacey S, Lee W, Michelfelder EC, Sr., Rempel GR, Silverman NH, Spray TL, Strasburger JF, Tworetzky W and Rychik J: Diagnosis and treatment of fetal cardiac disease: a scientific statement from the American Heart Association. Circulation 129(21): 2183-2242, 2014. PMID: 24763516. DOI: 10.1161/01.cir.0000437597.44550.5d

7 Hoffman JI and Kaplan S: The incidence of congenital heart disease. J Am Coll Cardiol 39(12): 1890-1900, 2002. PMID: 12084585. DOI: $10.1016 / \mathrm{s} 0735-1097(02) 01886-7$

8 Hoffman JI: Incidence of congenital heart disease: II. Prenatal incidence. Pediatr Cardiol 16(4): 155-165, 1995. PMID: 7567659. DOI: $10.1007 / \mathrm{BF} 00794186$

9 Gerlis LM: Cardiac malformations in spontaneous abortions. Int J Cardiol 7(1): 29-46, 1985. PMID: 4055133. DOI: 10.1016/ 0167-5273(85)90170-6

10 Christoffels VM, Burch JB and Moorman AF: Architectural plan for the heart: early patterning and delineation of the chambers and the nodes. Trends Cardiovasc Med 14(8): 301-307, 2004. PMID: 15596106. DOI: 10.1016/j.tcm.2004.09.002

11 Herghelegiu CG, Dragan I, Suciu N and Oprescu ND: A simplified protocol for congenital heart disease. 5TH Congr Rom Soc ULTRASOUND Obstet Gynecol, 298-302, 2017. 
12 Li Y, Hua Y, Fang J, Wang C, Qiao L, Wan C, Mu D and Zhou K: Performance of different scan protocols of fetal echocardiography in the diagnosis of fetal congenital heart disease: a systematic review and meta-analysis. PLoS One 8(6): e65484, 2013. PMID: 23750263. DOI: 10.1371/journal.pone.0065484

13 Herghelegiu CG, Duta SF, Neacsu A, Suciu N and Veduta A: Operator experience impact on the evaluation of still images of a first trimester cardiac assessment protocol. J Matern Neonatal Med 1-5, 2020. DOI: 10.1080/14767058.2020.1774873

14 Herghelegiu CG, Herghelegiu D, Neacşu A, Suciu ID and Suciu $\mathrm{N}$ : The 3-vessel view as a means of screening for conotruncal malformations. Obstet Ginecol (Bucur) LXVIII: 42-45, 2020. DOI: $10.26416 /$ ObsGin.68.1.2020.3037

15 International Society of Ultrasound in Obstetrics and Gynecology, Carvalho JS, Allan LD, Chaoui R, Copel JA, DeVore GR, Hecher K, Lee W, Munoz H, Paladini D, Tutschek $\mathrm{B}$ and Yagel S: ISUOG Practice Guidelines (updated): sonographic screening examination of the fetal heart. Ultrasound Obstet Gynecol 41(3): 348-359, 2013. PMID: 23460196. DOI: 10.1002/uog.12403

16 Quarello E, Lafouge A, Fries N and Salomon LJ: Basic heart examination: feasibility study of first-trimester systematic simplified fetal echocardiography. Ultrasound Obstet Gynecol 49(2): 224-230, 2017. PMID: 26799640. DOI: 10.1002/uog. 15866

17 Herghelegiu CG, Bacalbaşa N and Oprescu ND: Differential diagnosis between double outlet right ventricle and transposition of great arteries. 5TH Congr Rom Soc ULTRASOUND Obstet Gynecol, 294-297, 2017.

18 Herghelegiu CG, Ciutacu LA, Fetecau AC, Ioan RG, Neacsu A and Daniela Nuti: Congenitally corrected transposition of the great arteries - an apparently "benign" condition. Publons. Proc 6TH Congr ULTRASOUND Soc Obstet Gynecol, 2018.
19 Clur SA and Bilardo CM: Early detection of fetal cardiac abnormalities: how effective is it and how should we manage these patients? Prenat Diagn 34(13): 1235-1245, 2014. PMID: 25052917. DOI: $10.1002 /$ pd.4466

20 Perde F, Herghelegiu CG, Iosifescu AG, Crîngu I, Luca L and Dragu M: Pulmonary artery aneurysm in a marfanoid adult patient with unoperated functional single ventricle and levotransposition of the great arteries. Rom J Leg Med 26: 363-368, 2018. DOI: $10.4323 / \mathrm{rj} 1 \mathrm{~m} .2018 .363$

21 Wiechec M, Knafel A and Nocun A: Prenatal detection of congenital heart defects at the 11- to 13-week scan using a simple color Doppler protocol including the 4-chamber and 3vessel and trachea views. J Ultrasound Med 34(4): 585-594, 2015. PMID: 25792573. DOI: 10.7863/ultra.34.4.585

22 Weiner Z, Lorber A and Shalev E: Diagnosis of congenital cardiac defects between 11 and 14 weeks' gestation in high-risk patients. J Ultrasound Med 21(1): 23-29, 2002. PMID: 11794398. DOI: 10.7863/jum.2002.21.1.23

23 Volpe P, Ubaldo P, Volpe N, Campobasso G, De R, V, Tempesta A, Volpe G and Rembouskos G: Fetal cardiac evaluation at 1114 weeks by experienced obstetricians in a low-risk population. Prenat Diagn 31(11): 1054-1061, 2011. PMID: 21800333. DOI: $10.1002 / \mathrm{pd} .2831$
Received September 13, 2020

Revised September 25, 2020

Accepted September 30, 2020 